\title{
Normalisation of abnormal coronary fractional flow reserve associated with myocardial bridging using an intracoronary stent
}

\author{
B D Prendergast, F Kerr, I R Starkey
}

\begin{abstract}
Although intracoronary stenting procedures have been advocated for the successful treatment of myocardial ischaemia associated with myocardial bridging, the physiological rationale for this approach remains unexplored. The case of a 70 year old man with symptoms of cardiac ischaemia associated with a left anterior descending coronary artery bridge is described, where use of an intracoronary stent abolished the angiographic abnormalities and also restituted pronounced abnormalities of coronary fractional flow reserve.
\end{abstract}

(Heart 2000;83:705-707)

Keywords: angioplasty; myocardial bridge; coronary flow reserve; stent

Myocardial bridging, defined as the intramural course of a major epicardial coronary artery, is a relatively common finding at pathological examination, with a reported incidence ranging from $15-85 \% .{ }^{1}$ Identification in vivo, however,

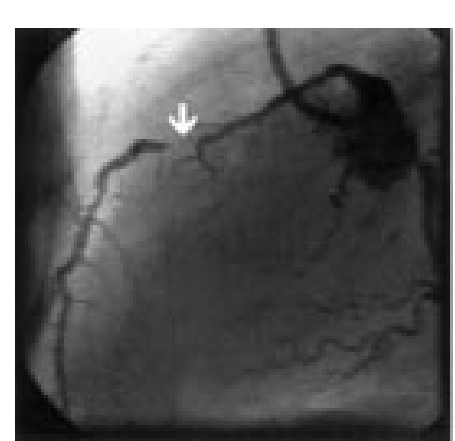

A
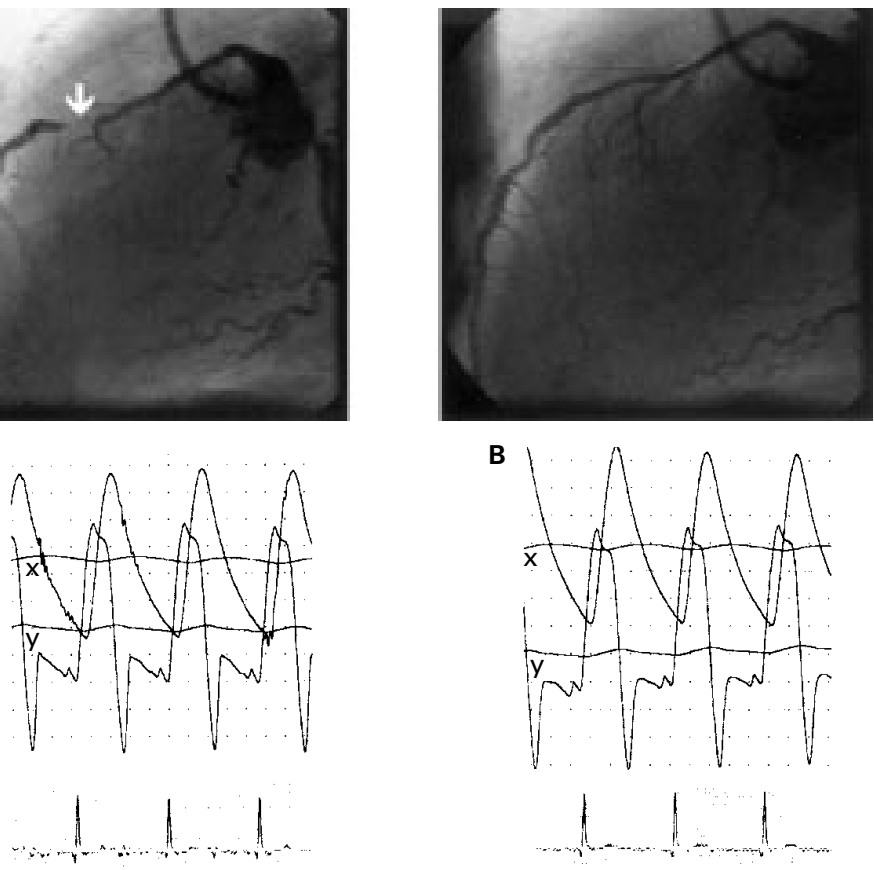

B

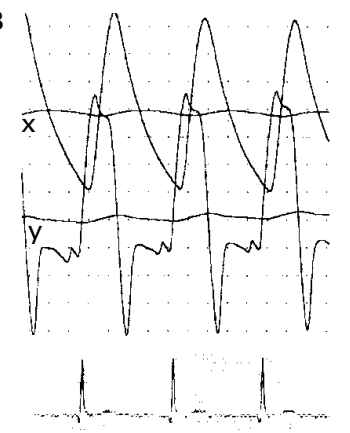

Figure 1 Top: Angiographic appearances of the left anterior descending coronary artery (ateral projection) in systole (left) and diastole (right). Myocardial bridging was associated with complete obliteration of the arterial lumen during systole (arrow). Bottom:

Simultaneous pressure recordings (phasic and mean) obtained from the right femoral artery and the left anterior descending coronary artery distal to the site of myocardial bridging, before (A) and after (B) administration of intracoronary adenosine $(20 \mu \mathrm{g})$. Coronary fractional flow reserve, expressed as the ratio of mean coronary arterial pressure (y) to mean aortic pressure $(x)$, was significantly reduced $(0.65$, normal $>0.75)$ following adenosine, confirming the functional significance of the bridged segment. at the time of angiography is much less frequent, with a reported incidence of $0.5-2.5 \%,{ }^{2}$ the left anterior descending coronary artery (LAD) being the most frequently affected vessel. Although the condition is generally considered benign, it has been associated with myocardial ischaemia, ventricular tachycardia, conduction disturbances, myocardial infarction, and sudden death. ${ }^{3}$ Several case reports have described intracoronary stenting procedures for the successful treatment of myocardial ischaemia associated with myocardial bridging, although the physiological rationale for this approach remains relatively unexplored. We herein describe a case where use of an intracoronary stent abolished the angiographic abnormalities and ischaemic symptoms associated with an LAD bridge, and also restituted pronounced abnormalities of coronary fractional flow reserve.

\section{Case report}

A 70 year old male non-smoker presented with an eight year history of exertional angina. There was a preceding history of hypertension but no myocardial infarction. The resting ECG was normal but during exercise testing he developed typical symptoms after four minutes of the Bruce protocol associated with an attenuated haemodynamic response and pronounced ST segment depression in both inferior and anterolateral leads. Treatment with a $\beta$ blocker, a long acting nitrate, and aspirin was instigated and he was referred for further investigation.

Selective coronary angiography showed generalised irregularity of the dominant right coronary artery but no focal stenosis. The left circumflex contained a severe proximal stenosis and the LAD demonstrated striking muscle bridging of approximately $20 \mathrm{~mm}$ length in its mid third with complete luminal obliteration during systole and normal appearances in diastole (fig 1). Left ventricular function was normal. Although the muscular bridge was likely to be of longstanding, it appeared that both "lesions" were of probable haemodynamic significance. After some discussion, it was therefore elected to proceed to circumflex angioplasty followed by a further procedure to the bridged segment of the LAD if symptoms and/or objective evidence of reversible myocardial ischaemia persisted.

Initial angioplasty to the left circumflex was performed using a $2.5 \times 20 \mathrm{~mm}$ Omnipass balloon (Cordis Europa) supplemented by deployment of a $3 \times 35 \mathrm{~mm}$ ACS Multilink Duet stent (Guidant Europa) to cover two 
areas of localised dissection. An excellent angiographic result was obtained and the patient made an uneventful recovery with no symptoms at follow up four months later. During exercise testing, he was able to complete seven minutes of the Bruce protocol without developing angina, but during exercise developed a progressive fall in systolic blood pressure from 140 to $110 \mathrm{~mm} \mathrm{Hg}$ associated with significant ST segment depression in the anterolateral chest leads, suggesting silent ischaemia. There were no ECG changes in the inferior leads.

Further angiography confirmed that the circumflex stent was patent with normal coronary flow. Measurements of LAD intracoronary pressure were then obtained using a fibreoptic pressure microtransducer incorporated into the distal segment of an 0.014 inch guidewire (Radi Medical Systems). ${ }^{4}$ These confirmed a significant pressure fall across the bridged segment with a reduced coronary fractional flow reserve of 0.65 (expressed as the ratio of mean coronary arterial pressure:mean aortic pressure, normal $>0.75$ ), measured following the administration of intracoronary adenosine $(20 \mu \mathrm{g})$ as a maximum hyperaemic stimulus (fig 1). ${ }^{4}$ An elective primary stent procedure was therefore performed using a $3 \times 25 \mathrm{~mm}$ ACS Multilink Duet stent (Guidant Europa) followed by high pressure expansion to 20 atm using a $3 \times 20 \mathrm{~mm}$ Tacker balloon (Cordis Europa). A residual dissection flap with associated localised stenosis was noted distal to the stent and dilated initially with the same Tacker balloon, followed by deployment of a $3 \times 13 \mathrm{~mm}$ Multilink stent. Further high pressure balloon expansion (maximum 22 atm) was applied to this stent and the short segment of stent overlap. Once more an excellent angiographic result was obtained and measurements

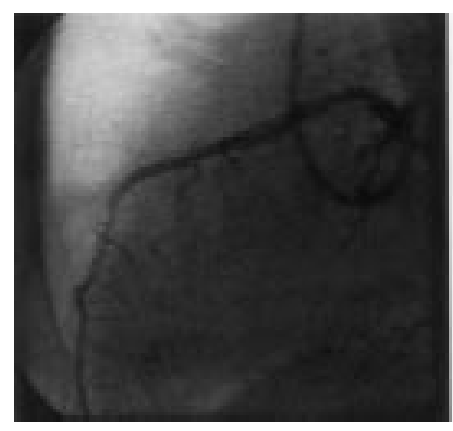

A

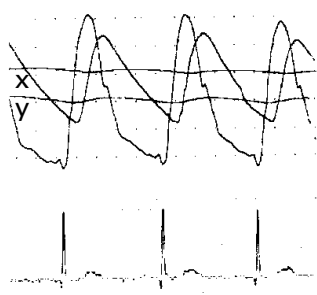

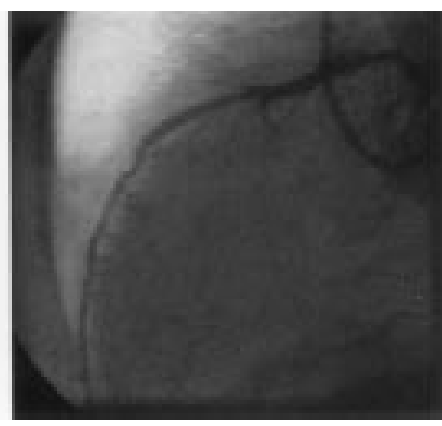

B

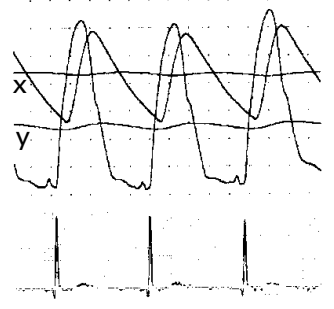

Figure 2 Angiographic appearances and simultaneous pressure recordings obtained following successful balloon angioplasty and stent deployment (format as for fig 1). Note the approximation of the mean pressure recordings with minimal change in response to intracoronary adenosine, confirming an improvement in normal fractional flow reserve, and restoration of a morphologically normal pressure trace distal to the treated segment. of intracoronary pressure confirmed an increase in coronary fractional flow reserve to 0.79 (fig 2). The patient made an uneventful recovery. Four months later, however, exertional angina returned and repeat angiography showed diffuse restenosis within the LAD stent, while the circumflex stent remained patent. An excellent angiographic result was obtained following further balloon angioplasty with no complications.

\section{Discussion}

Although myocardial bridging is a relatively common condition, controversy exists concerning its clinical and prognostic relevance, and the appropriate therapeutic approach for symptomatic patients with the anomaly. ${ }^{1}$ Furthermore, available treatment strategies have not been systematically evaluated, mainly because of incomplete understanding of the underlying haemodynamic mechanisms of ischaemia.

Recent quantative angiographic and intravascular ultrasound studies in patients with symptomatic myocardial bridging have showed that vessel compression is not limited to systole, causing the angiographic "milking" effect, but also persists into diastole, thereby affecting the predominant phase of coronary perfusion. ${ }^{5}$ In a recent detailed study, Klues and colleagues reported 12 patients with symptomatic myocardial bridges in whom coronary haemodynamics were assessed using quantitative coronary angiography combined with measurements of coronary flow velocity, coronary flow reserve, and intracoronary pressure. Coronary haemodynamics were characterised by phasic systolic vessel compression with a localised peak pressure, persistent diastolic diameter reduction, increased blood flow velocities, retrograde flow, and reduced flow reserve. ${ }^{6}$

On the basis of these observations, three therapeutic strategies may be of potential use: pharmacological intervention, surgery (either surgical myotomy or bypass grafting) or percutaneous angioplasty with stent deployment.

Negative inotropic agents, particularly $\beta$ blockers, may reduce systemic and intramural pressures and thereby external vessel compression. The accompanying negative chronotropic effect also prolongs diastole and thereby improves coronary perfusion. However, although the efficacy of this approach has been demonstrated in vivo, not all patients achieve persistent symptomatic relief. ${ }^{5}$

Until recently, surgical myotomy was considered the treatment of choice for patients with persistent symptoms despite medical treatment. Although cleavage of the bridging muscle fibres eliminates the underlying compression, deep incision of the ventricular wall is frequently necessary, with associated risk of mural aneurysm and scar formation with subsequent recurrent extrinsic vessel compression. Furthermore, it may be difficult to justify the small but tangible risk of surgery for the treatment of what is commonly single vessel disease. 
In view of these considerations, intracoronary stent implantation has been proposed as the optimal treatment for patients with symptomatic myocardial bridging, allowing internal stabilisation of the coronary artery lumen against external compression, thereby eliminating the abnormal pressure and flow phenomena. Several case reports have described a successful outcome using this approach with direct or indirect evidence for the improvement of underlying myocardial ischaemia. ${ }^{7-9}$ Consistent with the present report, Klues and colleagues demonstrated immediate normalisation of the flow velocity profile, lumen diameters, intracoronary pressures, and coronary flow reserve in three patients treated with an intracoronary stent. ${ }^{6}$ They also chose to use a Multilink stent because of its high flexibility in combination with the ability to withstand external compressive forces.

In our case, the use of a "pressure wire" allowed direct examination of the abnormal intracoronary pressure distal to the bridged segment associated with an abnormal coronary fractional flow reserve, confirming the functional significance of the myocardial bridge. Re-examination of the distal intracoronary pressure and fractional flow reserve following stent implantation confirmed an improved functional result from intervention. Although current recommendations suggest an optimal fractional flow reserve of $>0.94$ following stent deployment, ${ }^{10}$ we felt that the suboptimal result of 0.79 was related to diffuse distal disease rather than ineffective stent deployment. Whether extravascular compression caused by the presence of myocardial bridging, or poor distal run off, contributed to the subsequent development of in-stent restenosis remains unclear. In the study by Klues and colleagues, follow up assessment using angiography and intravascular ultrasound seven weeks following stent deployment revealed no evidence of in-stent restenosis in three patients studied. ${ }^{6}$ However, we are unaware of any longer term follow up data in patients who have undergone stent deployment for the treatment of myocardial bridging.

Stent implantation therefore represents an effective treatment strategy for symptomatic myocardial bridging. The present report provides further support for the efficacy of this approach, and underlines the importance of physiological assessment, both during initial diagnosis and in confirming the response to successful stent deployment. Larger long term studies remain necessary, however, to evaluate the incidence and degree of in-stent restenosis, the stability of stent geometry, and clinical improvement.

1 Angelini P, Trivellato M, Donis J, et al. Myocardial bridges: a review. Prog Cardiovasc Dis 1983;26:75-88.

2 Ishimori T, Raizner AF, Chabine RA, et al. Myocardial bridges in man: clinical correlations and angiographic accentuation with nitroglycerin. Cathet Cardiovasc Diagn 1977;3:59-65.

3 Noble J, Bourassa MG, Peticlerc R, et al. Myocardial bridging and milking effect of the left anterior descending coronary artery: normal variant or obstruction? Am f Cardiol 1976;37:993-9.

4 Pijls NHJ, de Bruyne B, Peels K, et al. Measurement of fractional flow reserve to assess the functional severity of

corar Schwarz ER, Klues HG, vom Dahl J, et al. Functional, ngiographic and intracoronary Doppler flow characterismyocardial bridging: effect of acute intravenous beta blocker medication. f Am Coll Cardiol 1996;27:1637-45.

6 Klues HG, Schwarz ER, vom Dahl J, et al. Disturbed intracoronary hemodynamics in myocardial bridging. Early normalization by intracoronary stent placement. Circulation 1997;96:2905-13.

7 Stables RH, Knight CJ, McNeill JG, et al. Coronary stenting in the management of myocardial ischaemia caused by myocardial bridging. Br Heart f 1995;74:90-2.

8 Smith SC, Taber MT, Robiolio PA, et al. Acute myocardial infarction caused by a myocardial bridge treated with intracoronary stenting. Cathet Cardiovasc Diagn 1997;42:20912.

9 Bayés A, Martí V, Augé JM. Coronary stenting for symptomatic myocardial bridging. Heart 1998;80:102-3.

10 Pijls NHJ, de Bruyne BDE. Fractional flow reserve for evaluation of coronary interventions. Coronary pressure. Dordrecht: Kluwer Academic Publishers, 1997:261-71. 